\title{
DOUBLING BEAM INTENSITY UNLOCKS RARE OPPORTUNITIES FOR DISCOVERY AT FERMI NATIONAL ACCELERATOR LABORATORY
}

\author{
At Fermi National Accelerator Laboratory, upgrading the 40-year-old RF cavities in the \\ Booster synchrotron will provide a twofold improvement in proton throughput for high-intensity \\ particle physics experiments that could lead to breakthrough discoveries about the universe.
}

\section{BY JENNIFER A. SEGUI, COMSOL, INC.}

PARTICLE ACCELERATORS SUCH as the Booster synchrotron at the Fermi National Accelerator Laboratory (FNAL) produce high-intensity proton beams for particle physics experiments that can ultimately reveal the secrets of the universe. High-intensity proton beams are required by experiments at the "intensity frontier" of particle physics research, where the availability of more particles improves the chances of observing extremely rare physical processes. In addition to their central role in particle physics experiments, particle accelerators have found widespread use in industrial, nuclear, environmental, and medical applications.

RF cavities are essential components of particle accelerators that, depending on the design, can perform multiple functions, including bunching, focusing, decelerating, and accelerating a beam of charged particles. Engineers Mohamed Awida Hassan and Timergali Khabiboulline, both from the Superconductivity and Radiofrequency Development Department of FNAL's Technical Division, are working in collaboration with John Reid from the

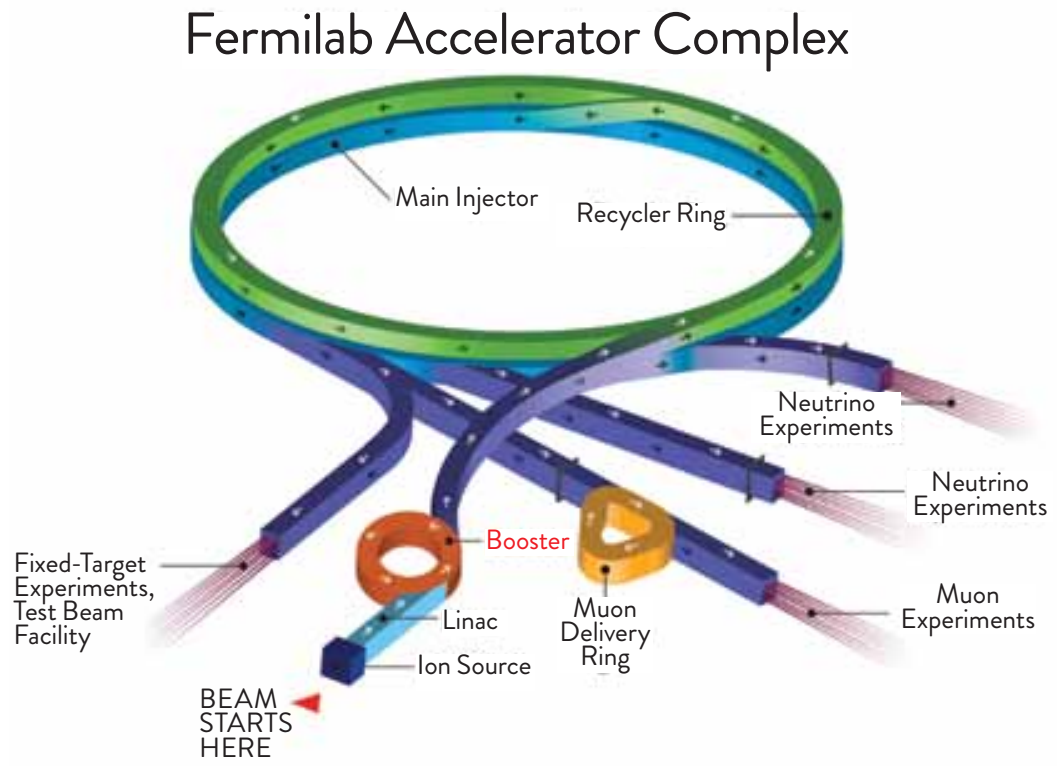

FIGURE 1: The FNAL accelerator chain showing the location of the Booster synchrotron.

Accelerator Division to model the RF cavities required for upgrading the 40-year old Booster synchrotron. Reid leads the rather complicated process to refurbish, test, and qualify the upgraded RF cavities.

"In our work, we demonstrate the early-stage feasibility of the upgraded RF cavities to sustain an increased repetition rate of the $\mathrm{RF}$ field required to produce proton beams at double the current intensity," says
Hassan. "We are using both multiphysics simulation and physical measurements, provided by our colleagues in the Accelerator Division, to evaluate the RF, thermal, and mechanical properties of the Booster RF cavities."

\section{POWERING PARTICLE PHYSICS RESEARCH} FNAL IS CURRENTLY enacting its Proton Improvement Plan (PIP), under the leadership of William Pellico and Robert Zwaska. The plan calls for facility upgrades in order to double the beam throughput and 

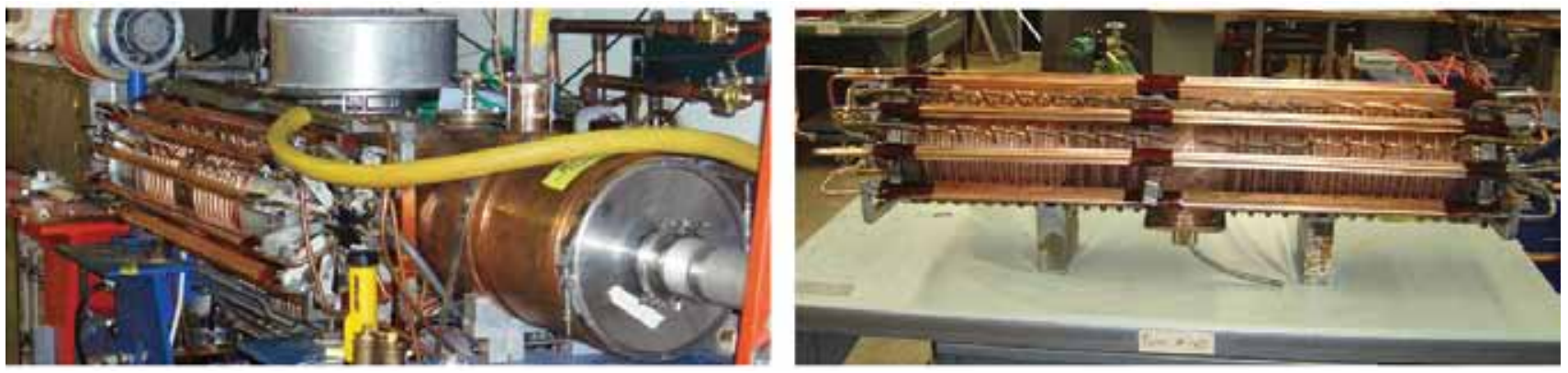

FIGURE 2: At left, a photograph of a copper ferrite-tuned RF cavity from FNAL's Booster synchrotron. Above, a ferrite tuner.

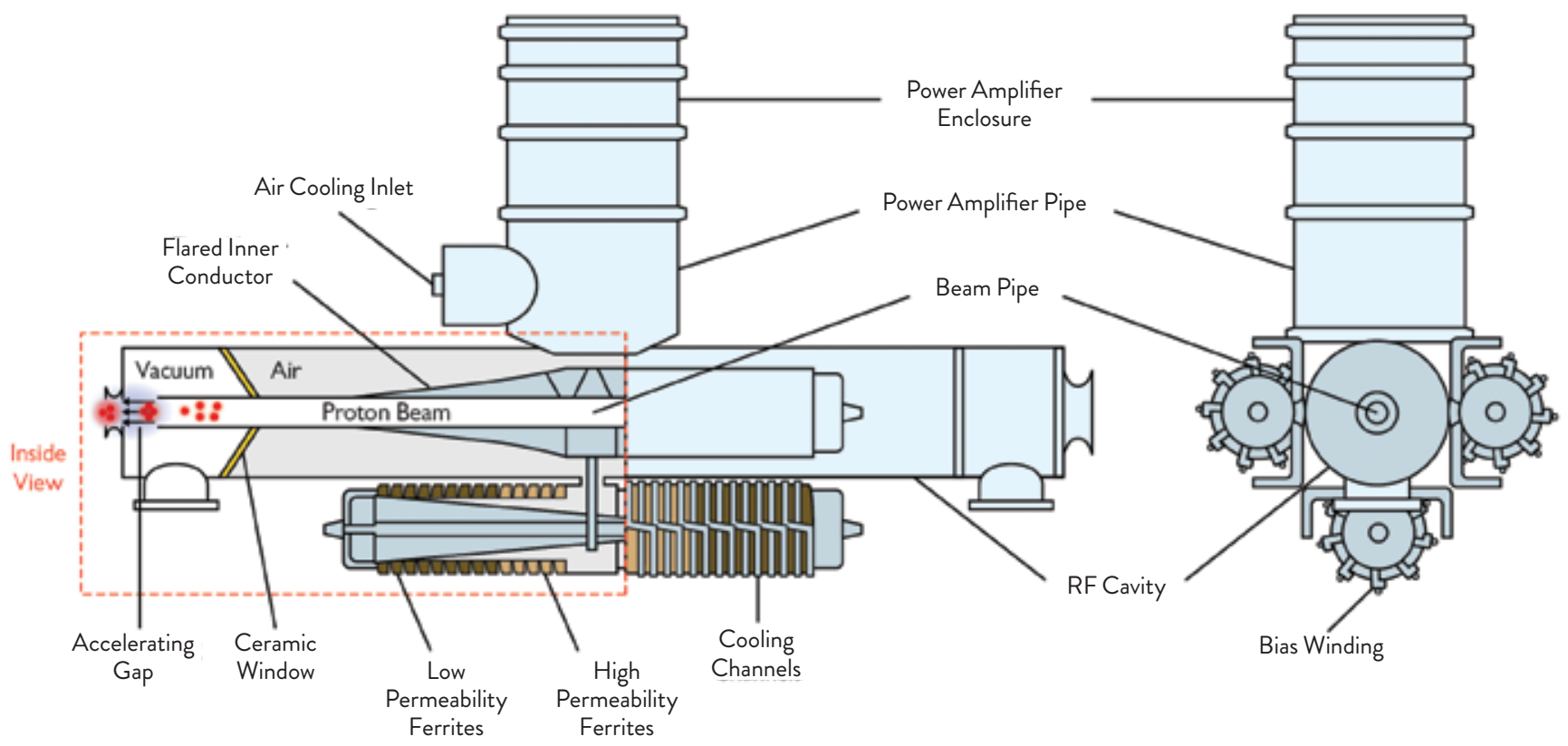

FIGURE 3: Front- and side-view drawings of a Booster RF cavity with three ferrite tuners and a tetrode power amplifier. The side-view drawing shows the high- and low-permeability ferrites, including the cooling channels required to prevent overheating. The ferrites are enclosed in a copper tube that has been eliminated in this drawing in order to expose more detail.

modernize the particle accelerators. A schematic of the accelerator chain at FNAL is shown in Figure 1. The Booster synchrotron, a cyclic particle accelerator and intermediate stage in the particle accelerator chain, is highlighted in the figure. Located about 20 feet below ground, the Booster uses magnetic fields to bend the proton beam in a circular path while 19 ferrite-tuned RF cavities accelerate the protons to 20 times their initial energy when first arriving at the Booster. The protons are transferred to the Main Injector synchro- tron, where they are further accelerated, and then directed to multiple underground beam lines. Protons in the underground beam lines interact with neutrino production targets, experimental target materials, or detectors as part of testing.

\section{THE WORKHORSE OF THE BOOSTER SYNCHROTRON}

ONE OF THE remaining challenges of the Proton Improvement Plan is upgrading the $\mathrm{RF}$ cavities of the Booster synchrotron so they can handle the higher-intensity beams. A photograph of a Booster RF cavity is shown in Figure 2. The Booster RF cavities are half-wave resonators that generate an oscillating electromagnetic field to accelerate protons along the central beam pipe. Each RF cavity is loaded with three coaxial ferrite tuners placed at 90-degree intervals to achieve sufficiently low inductance. 


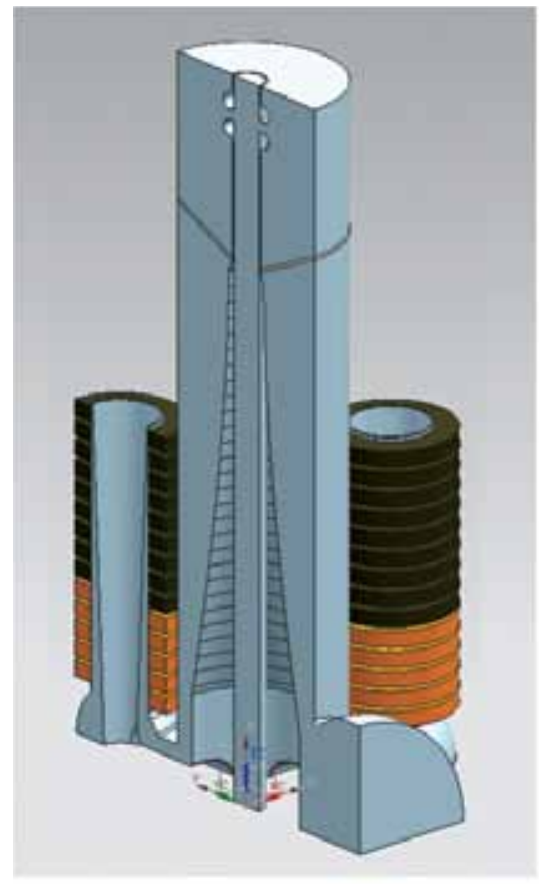

FIGURE 4: RF model geometry for the Booster RF cavity with ferrite tuners. Onequarter of the symmetric cavity design was modeled and imported into COMSOL.

In the fourth position, a tetrode power amplifier supplies the RF signal. Sideand front-view drawings of a Booster RF cavity are shown in Figure 3.

The ferrite-tuned RF cavities are designed with a specific size and shape in order to resonate at a certain frequency and with a 40 percent tuning range. As protons cycle through the Booster, the frequency is gradually increased by biasing the ferrite tuners to get the particles up to the target energy. The operating frequency range of the RF cavities will not change as part of the PIP. Parameters such as the accelerating voltage and beam repetition rate, which governs how often particle beams are produced and sent through the accelerator chain, do need to increase, however.

\section{SIMULATION QUANTIFIES RF HEATING} OPERATING THE BOOSTER RF cavities at the higher repetition rate and

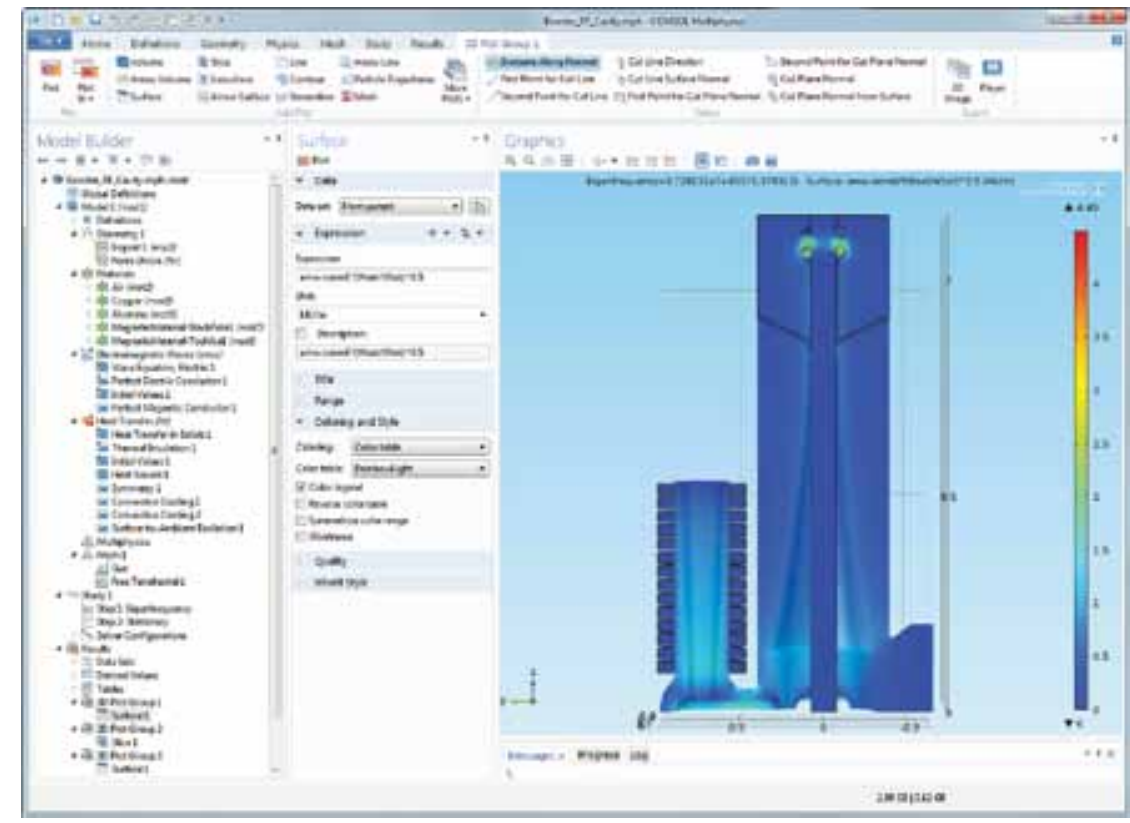

FIGURE 5: This COMSOL Desktop $®$ image shows model setup and analysis for a multiphysics model of an RF cavity. The geometry, materials, physics, and study are defined in the Model Builder window at left. A surface plot of the electric field on the RF cavity and tuner is displayed in the Graphics window. RF analysis is initially conducted to capture the electric and magnetic fields that will be used later as sources of heating in the thermal analysis. The electric field distribution was also investigated to ensure that breakdown will not occur near the high-field regions in air or under vacuum.

accelerating voltage is necessary in order to increase the overall efficiency of the particle accelerators and double the hourly proton yield. An increase in the power dissipated in the RF cavities is projected, however, which could lead to overheating. Additional thermal stress in the cavity and tuners could potentially reduce their lifetime and produce an unreliable proton yield. Better cooling may be required to ensure stable longterm proton production at the desired rate. The current cooling mechanism uses water circulating in pipes surrounding the cavities in addition to fans that generate a cooling air flow.

Hassan and Khabiboulline are evaluating the Booster RF cavities to estimate the cooling requirements at the increased repetition rate and accelerating voltage. Physical measurements of temperature in the RF cavity and tuner can be difficult to acquire and are often inaccurate. Multiphysics simulations were used in conjunction with experiments to develop a model of the RF cavity that could be used to evaluate its RF, mechanical, and thermal properties. The model was set up in COMSOL Multiphysics ${ }^{\circledR}$, where one-quarter of the actual geometry was imported from an $\mathrm{SAT}^{\circledR}$ file that was created in a separate CAD program. The imported model geometry is shown in Figure 4 and includes the cavity and tuners. "We chose to simulate only part of the symmetric design to reduce the computational complexity and time required to solve the model," says Hassan. Perfect magnetic conductor (PMC) boundary conditions were enforced along the symmetry planes while the perfect electric conductor (PEC) boundary condition was enforced on all other boundaries in the RF model.

The materials, physics, and study were set up as shown in the model tree in Figure 5. The copper material for the walls was defined using the built-in material properties available in the Material Library. The properties of the ferrite material for the tuners were custom- 


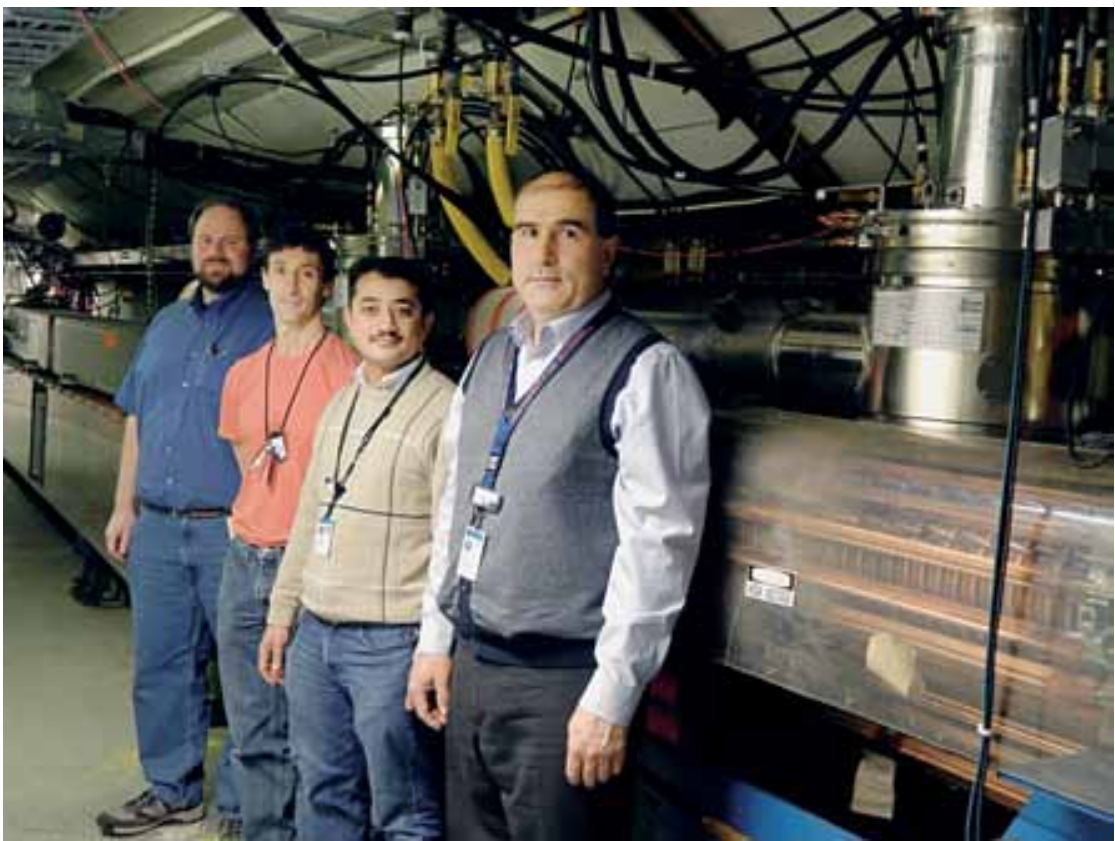

From left to right, the engineers behind the Proton Improvement Plan and RF cavity simulations: Robert Zwaska, PIP deputy leader; William Pellico, PIP leader; Mohamed Hassan, senior RF engineer; and Timergali Khabiboulline, RF Group leader. They are pictured in the Booster synchrotron tunnel at FNAL, next to a ferrite-tuned RF cavity. John Reid, not pictured, is the RF Group Leader from the Accelerator Division.

defined. Initially, the electromagnetic problem was evaluated to solve for the electric and magnetic fields. Electromagnetic losses in the ferrite and resistive losses along the cavity surface were used as heat sources for solving the heat transfer problem. The cooling mechanism was incorporated into the model by applying the convective heating boundary condition to the outer walls of the tuner. The model was validated by comparing the measured quality factor $(\mathrm{Q})$ of the RF cavity with the quality factor computed in the $\mathrm{COMSOL}^{\circledR}$ environment.

Thermal analysis was performed to show the effect of increasing the repetition rate and accelerating voltage on the operating temperature of the tuners. The results shown in Figure 6 are for an accelerating voltage of 55 kilovolts and repetition rate of 7 hertz where a temperature maximum of $65^{\circ} \mathrm{C}$ was observed in the tuners. The accelerating voltage was held constant at 55 kilovolts, while the repeti- tion rate was increased from 7 to 15 hertz. The analysis showed that this approximate doubling of the repetition rate could cause the operating temperature of the tuners to increase by more than $30^{\circ} \mathrm{C}$. A further increase in the accelerating voltage to $60 \mathrm{kV}$ while operating at the $15-\mathrm{Hz}$ repetition rate could cause the operating temperature to increase by another $10^{\circ} \mathrm{C}$. The power dissipated in the RF cavity and tuners increased from $16.6 \mathrm{~kW}$ at $55 \mathrm{kV}$ and $7-\mathrm{Hz}$ repetition rate to $39.1 \mathrm{~kW}$ at $60 \mathrm{kV}$ and $15-\mathrm{Hz}$ repetition rate.

\section{॥ ENSURING SMOOTH OPERATION THROUGH 2025} BASED ON THE simulation results, Hassan confirms that "the cooling mechanism will need to be upgraded along with the cavities to handle the increased repetition rate and accelerating voltage through 2025 as called for in the Proton Improvement Plan." Increasing the airflow will be one of the first adjustments made, although adding more pipes, fur-

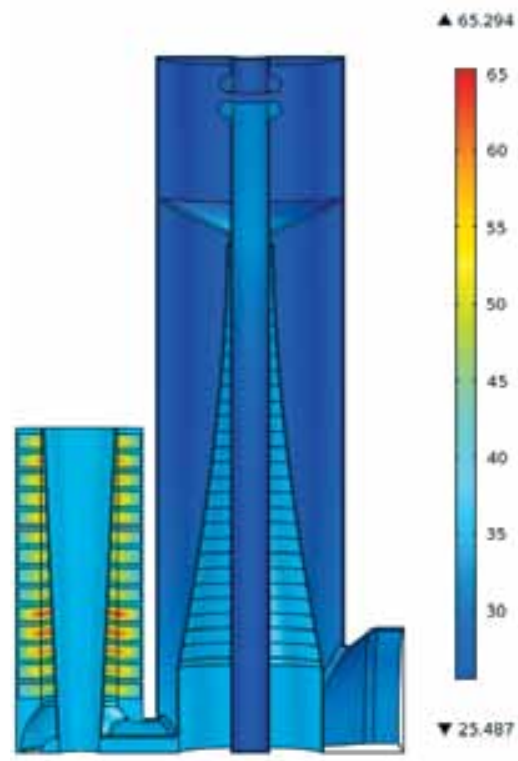

FIGURE 6: A surface plot of temperature is shown from the thermal analysis of an FNAL Booster RF cavity at 55-kV accelerating voltage and $7-\mathrm{Hz}$ repetition rate.

ther reducing the water temperature, and experimenting with the water flow rate are all possibilities. The RF cavity model will be expanded in the future to include air and pipe flow so that the geometry and cooling mechanism more closely represents that of the actual RF cavity.

In the extreme environment of the Booster synchrotron, radiation hazards and high temperatures make upgrading the RF cavities a challenge. Simulation results are being used to facilitate design decisions with regard to the cooling mechanism to help reduce the time, risks, and expense associated with the upgrade and continued use of the RF cavities. Successfully implementing the improved cooling system will aid in keeping the unique RF cavities of the Booster synchrotron not only operational, but generating more high-energy protons than ever and through their sixtieth year. () 